Portland State University

PDXScholar

\title{
Understanding Support Network Capacity During the Transition from Foster Care: Youth-identified Barriers, Facilitators, and Enhancement Strategies
}

\author{
Jared Best \\ Portland State University, jibest@pdx.edu \\ Jennifer E. Blakeslee \\ Portland State University, jblakes@pdx.edu
}

Follow this and additional works at: https://pdxscholar.library.pdx.edu/socwork_fac

Part of the Family, Life Course, and Society Commons, and the Social Work Commons Let us know how access to this document benefits you.

\section{Citation Details}

Best, Jared and Blakeslee, Jennifer E., "Understanding Support Network Capacity During the Transition from Foster Care: Youth-identified Barriers, Facilitators, and Enhancement Strategies" (2019). Social Work Faculty Publications and Presentations. 281.

https://pdxscholar.library.pdx.edu/socwork_fac/281

This Post-Print is brought to you for free and open access. It has been accepted for inclusion in Social Work Faculty Publications and Presentations by an authorized administrator of PDXScholar. Please contact us if we can make this document more accessible: pdxscholar@pdx.edu. 


\section{Accepted Manuscript}

Understanding support network capacity during the transition from foster care: Youth-identified barriers, facilitators, and enhancement strategies

Jennifer E. Blakeslee, Jared I. Best

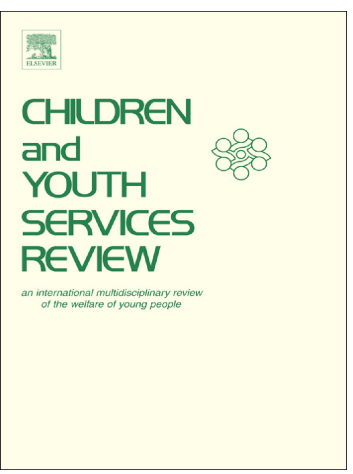

PII: $\quad$ S0190-7409(18)30683-2

DOI: $\quad$ https://doi.org/10.1016/j.childyouth.2018.11.049

Reference: $\quad$ CYSR 4109

To appear in: $\quad$ Children and Youth Services Review

Received date: $\quad 12$ August 2018

Revised date: $\quad 26$ November 2018

Accepted date: $\quad 26$ November 2018

Please cite this article as: Jennifer E. Blakeslee, Jared I. Best, Understanding support network capacity during the transition from foster care: Youth-identified barriers, facilitators, and enhancement strategies. Cysr (2018), https://doi.org/10.1016/ j.childyouth.2018.11.049

This is a PDF file of an unedited manuscript that has been accepted for publication. As a service to our customers we are providing this early version of the manuscript. The manuscript will undergo copyediting, typesetting, and review of the resulting proof before it is published in its final form. Please note that during the production process errors may be discovered which could affect the content, and all legal disclaimers that apply to the journal pertain. 
Understanding support network capacity during the transition from foster care: Youth-identified

barriers, facilitators, and enhancement strategies

Jennifer E. Blakeslee, Jared I. Best

Portland State University

Corresponding author:

Jennifer Blakeslee

jblakes@pdx.edu

Portland State University

School of Social Work, Regional Research Institute

1600 SW $4^{\text {th }}$ Avenue, Suite $\# 900$

Portland, OR 97201

503-725-8389 


\begin{abstract}
This study explores how foster care experiences can impact support network functionality as young people exit the foster care system. This can be conceptualized as a function of both network member capacity to provide adequate support to address young adult needs, and network stability, which reflects cohesion within and across relationships to facilitate consistent support over time. We conducted support network mapping and semi-structured interviews with youth in foster care aged 16$20(\mathrm{~N}=22)$ and used theoretical thematic analysis to explore support barriers and facilitators in relation to the organizing concepts of support capacity and network stability. Overall, support capacity was limited by interpersonal difficulties inhibiting the presence and supportiveness of some network members (including family members, informal peer and community-based connections, and caseworkers), whereas network stability facilitated multidimensional support through strong and interconnected relationships with caregivers and service providers. Emergent network patterns reflected distinct subgroups of more and less functional support networks, and strategies for network enhancement focus on promoting youth-directed services and support, developing youth skills and opportunities to invest in informal relationships, and using network assessment to identify unmet support needs. Findings advance a framework for understanding how foster care impacts support network characteristics, and inform ongoing efforts to address resulting limitations through services and programming.
\end{abstract}




\section{Introduction}

Of the nearly 438,000 children and youth in foster care in the United States, almost 46,000 are aged 16 or older, and over 20,000 "age out" of many child welfare services between ages 18-20 every year (US Department of Health and Human Services, 2017). These young people became wards of the state due to maltreatment at some point, and are expected to transition directly from foster placements (including foster homes, group homes, and congregate care) and into adulthood, having not been legally reunified with their family of origin, adopted, or placed under permanent guardianship, prior to reaching the age of majority. The research is clear that this population experiences relatively poor outcomes (see Gypen, Vanderfaeillie, De Maeyer, Belenger, \& Van Holen, 2017 for a recent review), including limited educational attainment and higher rates of homelessness, unemployment, criminal justice involvement, and mental health and substance abuse challenges (e.g., Brown, Courtney, \& McMillen, 2015; Courtney at al., 2018; Dworsky, Napolitano, \& Courtney, 2013; Havlicek, Garcia, \& Smith, 2013).

Importantly, although the consistent provision of multidimensional social support is recognized as an important resource during transitions to adulthood (IOM and NRC, 2013), there is consensus that such support is too often scarce for youth aging out of foster care (e.g., Avery, 2010; Goodkind, Schelbe, \& Shook, 2011; Rutman \& Hubbertstey, 2016). dolescent development is typically facilitated in the socioecological context of stable family, school, and community networks, which can ameliorate psychological and behavioral problems and promote well-being (Biglan, Flay, Embry, \& Sandler, 2012; Brooks, Magnusson, Spencer, \& Morgan, 2012; McPherson et al., 2013). Child welfare services are intended to support such development by connecting youth to comprehensive resources through a combination of services and informal support, including maintenance of ties to family and community of origin. Ideally, foster placement facilitates a network structure providing multidimensional support and resources, much as a typical family does (Coleman, 1988; Wellman \& Frank, 2001). Such family-based or 
"family-like" network functionality likely contributes to the relatively positive outcomes and functioning of subgroups of older foster youth in stable foster homes with relatives or long-term caretakers (Blakeslee, Kothari, McBeath, Sorenson, \& Bank, 2017; Cashmore \& Paxman, 2006; Daining \& DePanfilis, 2007; Keller, et al., 2007; Shpiegel \& Ocasio, 2015; Yates \& Grey, 2012).

However, for many young people in care, network-based social support is limited by the circumstances that led to initial foster placement, as well as ongoing disruption and disconnectionfrom caretakers and relatives, schools and peers, neighborhoods and communities-while in foster care. Research shows that there is a large subgroup of foster youth who experience repeated network disruption due to placement instability, specifically involving non-relative or group homes and residential treatment (e.g., McCoy, McMillen, \& Spitznagel, 2008; Keller et al., 2007; Wulcyn, Kogan, \& Harden, 2003). Repeated disruption can result in sparse networks, service disengagement, problematic behaviors, and social adjustment challenges (e.g., Perry, 2006; James, Landsverk, \& Slymen, 2004; Shook et al., 2011; Unrau, Seita, \& Putney, 2008), all of which may further impact the stability and capacity of their networks to provide support. Thus, many of these young people approach adulthood with exceptional challenges, and without a stable family-based network to address needs as the formal support of foster care case management recedes (Berzin, Singer, \& Hokanson, 2014; Munson, Lee, Miller, Cole, \& Nedelcu, 2013; Zinn, Palmer, \& Nam, 2017).

Research also consistently highlights the important role of non-parental adults in improving transition outcomes for older foster youth (Ahrens et al., 2011; Geenen \& Powers, 2007; Greeson \& Bowen, 2008; Gypen, et al., 2017; Munson \& McMillen, 2009; Thompson, Greeson, \& Brunsink, 2016), and studies focus on the presence of informal relationships to help bridge the expected shift from formal to informal support resources as services end (Greeson \& Thompson, 2017; Jones, 2013; Nesmith \& Christophersen, 2014; Singer, Berzin, \& Hokanson, 2016; Paulson \& Berg, 2016). Yet, long-term foster care leaves a subgroup of emancipating youth disconnected from informal relationships, disengaged 
from institutions and services, and disillusioned with the child welfare system (e.g., Keller et al., 2007), just as they are being encouraged by the child welfare system to actively participate in planning for their transition into independence. (For example, most young people in foster care over age 16 are eligible for federally-funded Independent Living Programs [ILPs], which focus on transition-related skill-building, although only about half of eligible youth access these services [Okpych, 2015]).

Importantly, research also shows that older youth in foster care are more likely to feel that it is useless, inadvisable, or risky to seek help from others, especially if they have had a history of multiple placements or are aging out of care (Seita, Day, Carrellas, \& Pugh, 2016). This kind of "survivalist selfreliance" (Samuels \& Pryce, 2008) is not uncommon when young people exit care lacking more normative experiences of social development and community integration (e.g., Pryce, Napolitano, \& Samuels, 2017). This has also recently been explored in terms of the effect of trauma-related mental health diagnoses (Morton, 2018) and the development of avoidant attachment styles (Okpych \& Courtney, 2018) specifically impacting post-secondary achievement among former foster youth.

To assess and address network-oriented support challenges before youth emancipate from care, child welfare service providers need to identify youth who may lack a family-based network, or who may struggle to develop supportive relationships, and/or who may be overly self-reliant based on prior lived experiences. We can measure foster youth networks in research, in terms of the presence of a range of types of supportive relationships and dimensions of support satisfaction and strain (e.g., Courtney et al., 2017), which provides comprehensive quantitative descriptions of youth connectedness. Another potentially fruitful approach is to explore support provision as a function limited by the structure and composition of the youth network as a whole, and as revealed through analysis of important network indicators. This approach can also be used to assess support network functionality in practice, and to guide strategies to facilitate support enhancement through service provision. This paper applies network 
theory to specifically explore barriers and facilitators of support network functionality, and to identify potential support network enhancement strategies from the perspective of youth in foster care.

\subsection{Support network enhancement}

Although increasing social support is not a new practice objective, we don't know enough about how to assess and enhance limited foster youth support networks as part of typical service provision. In previous research, the first author applied transdisciplinary knowledge about social network structure, network member composition, and relationship characteristics to better understand youth support networks. This included conceptualization of network-level dimensions of support capacity (indicated by size, diversity, range, etc.) and network stability (also called network cohesion) as particularly relevant to youth in foster care. Additionally, these indicators are measurable through systematic network mapping for both research purposes and practice-based assessment (Blakeslee, 2015; Blakeslee \& Keller, 2016). Using this framework, we can conceptualize support network functionality as being dependent on a combination of characteristics which reveal "emergent" properties of the network as a whole.

For example, support capacity is limited by network size, in terms of the number of people who can provide any support, as well as the range of social roles (e.g., parent figures, service providers) and domains (e.g., family, friends, school and work settings, community connections) represented by those network members, and whether this combination of people, roles, and domains results in an overall network that can provide adequate and comprehensive support to the youth as needed (Blakeslee, 2015). On the other hand, network stability reflects support consistency over time, as determined by the cohesiveness of relationships with and between members, which contributes to a family-like "core" network that can resist the disruption of support provision to youth when individual members fall away due to circumstance or conflict (Blakeslee \& Keller, 2016; Moody \& White, 2003). Here, we can assess interconnection, which provides structural stability (e.g., people who know each other can communicate about youth needs or help resolve conflict), as well as the presence of multidimensional support 
provision through one or more strong relationships between youth and the individual members, because stable relationships tend to provide comprehensive support (and vice versa).

\subsection{Current study}

This study applies these support network concepts as a theoretical framework to explore foster youth support network functionality, and to identify related enhancement strategies that can potentially improve the functionality of the network as a whole. We use a network mapping and semi-structured interview protocol developed to assess and explore presumed indicators of support network capacity and stability from the youth perspective. The current analysis specifically identifies youth perceptions of facilitators and barriers to support provision, as understood in relation to these broad network concepts. Lastly, we summarize identified strategies for support network enhancement from the perspective of the participants, and discuss these as relevant in developing new approaches to enhance both the capacity and stability of limited support networks before youth exit the child welfare system.

\section{METHODS}

\subsection{Sample description and recruitment}

Our sample includes 22 youth aged 16-20 (mean=17.68 years) who had an open Oregon child welfare and were eligible for foster placement. Twelve participants identified as female $(55 \%)$ and ten as male, and eight identified as part of the LGBTQ community. Participants described their own race and/or ethnicity with exactly half (11) who identified as White/Caucasian; six identified as "mixed," two as Black and/or African American, and three as another race/ethnicity. Over half of the sample was from Oregon's only metropolitan/urban area $(n=12,55 \%)$, while five were from mid-sized cities across the state, and five lived in more rural settings (determined by population density per square mile). 17 were currently involved in ILP services, and youth-reported time in foster care ranged from eight months to over ten years, with an average between four and five years. At the time of the interview, $55 \%(n=12)$ were living in a foster home (four of those were kin or biological families), and four were living in 
residential facilities, three were living with friends or friends' parents in uncertified foster homes, one lived with roommates, one lived in college student housing, and one was living in a homeless shelter.

The study protocol was approved by Portland State University's Institutional Review Board (IRB) and the Oregon Department of Human Services (DHS) external research committee. Study recruitment occurred in three purposive phases designed to include an exploratory sample of youth who were more and less connected to services, who were from metropolitan and more rural areas, and who lived in a variety of foster care placements, including foster family homes, group homes, and other supervised placements. First, we recruited at a statewide event for foster youth interested in post-secondary education or training; approximately 50 event attendees were told about the study, 25 interested youth provided contact information, we received caseworker consent for ten of these youth to participate, and then scheduled the interviews $(n=6)$. This strategy resulted in participants from both rural and metropolitan areas, although they can be considered relatively service-connected, given that they were attending an event hosted by the state ILP program. Next, to increase regional diversity, DHS generated a random sample of 50 youth in foster care across the state, and we contacted the caseworkers for these youth; four of the youth were removed from the list due to circumstance (e.g., case closing, youth on the run). If caseworkers consented, we contacted youth to request their participation ( $n=9)$; two caseworkers also referred additional youth living in the same foster home $(n=2)$. This second strategy resulted in a more diverse group of participants in terms of being less connected to services and not enrolled in ILP, and from a range of geographic areas and placement types (including three youth who were living with friends in placements that were not certified by DHS); however, though these participants came from a statewide list, response rate was low and this subgroup is not representative. Lastly, we recruited broadly from ILP providers across the state $(n=5)$, which resulted in two youth living in a semi-rural area and three who were in behavioral treatment facilities. Overall, approximately $43 \%$ of the caseworkers we contacted to recruit a specific youth responded and provided consent, and $53 \%$ of 
the youth who we contacted directly following caseworker approval agreed to participate. All study participants provided written consent and received a $\$ 20$ gift card to thank them for participating.

Given the range of sampling strategies employed, including the potential gatekeeping role played by caseworkers and ILP providers, we note that our sample is not representative of youth in foster care in general or in Oregon specifically. For example the proportion of youth connected to ILP (77\%) is much higher than recorded in representative samples of foster youth (e.g., Okpych, 2015), as is the number of LGBTQ-identified youth in our study (36\%, compared to $26 \%$ in Courtney et al., 2018), and we assume that non-respondent caseworkers, ILP providers, and young people differ in relevant ways from the successfully recruited sample (see limitations section for additional discussion). Rather, our findings reflect data from an exploratory sample of relatively diverse, but not representative, young people in care who were living in a range of foster placement types and more and less populated areas, and were responsive to direct recruitment for a one-time interview about their support networks.

\subsection{Data collection}

Data were collected using a support network map instrument and a semi-structured interview that referenced the network map throughout. The principal investigator and the doctoral student interviewer initially piloted the data collection protocol with two project interns; an undergraduate student intern completed the network map and interview protocol while another graduate student intern, who had lived experience of aging out of foster care, observed and gave feedback regarding the mapping activity and the follow-up interview questions. This process was used to revise the initial map instrument and interview protocol, which were further refined during the first phase of recruitment.

2.2.1. Support network mapping. The network map was adapted from earlier research using a similar instrument (Blakeslee, 2015; Blakeslee \& Keller, 2016) to generate network member names, types of support provided, and strength of relationships. The network map is not unlike other ecomapping tools used in research and practice, and was specifically developed to measure foster youth 
support networks. For this study, the map was adapted to be more youth-friendly and to capture more information on a single page, and was used primarily for the purpose of guiding the interview questions.

On each map, youth wrote the names or initials of people who had played a role in their lives in the past year within four broad domains, presented as quadrants on the page. The interviewer explained the domains as follows: Family includes anyone considered family, Friends generally includes peers and significant others, School/work typically includes teachers, supervisors, and/or coworkers, and Community often includes neighbors, people from church or other community groups, and service providers such as caseworkers, therapists or counselors, and/or ILP staff. Youth drew lines between their initials in the center of the map and the network members, with the line thickness indicating whether the relationship was strong, weak, or neutral. Strong ties were defined as people by whom the participant felt most supported, while weak ties could represent relationships that existed due to circumstance (e.g., another youth in a foster home), those where conflict was present, or where contact was infrequent. Neutral ties were neither especially strong nor weak. Youth then indicated the type(s) of support each person provided by drawing symbols (e.g., a heart, a star) shown in a legend next to the map. These included emotional support (e.g. people with whom participants shared personal news, someone to lean on during difficult times), concrete support (e.g. a place to sleep, money, a ride), and informational (such as academic advising, getting a driver's license, writing a resume). Lastly youth circled individuals with whom they had regular contact (at least weekly), and drew interconnecting lines between people who also knew each other.

Although the maps themselves were not analyzed for this paper, they were quantitatively coded as described in Blakeslee (2015) for the purpose of sample description. On average, participants named 15 people as having played a supportive role in their lives in the previous year (mean=15.0, SD=6.5), with about six "core" members providing more regular support $(m=6.4, S D=3.5)$. Family ties were most common ( $m=5.18, S D=3.28)$, followed by friends, community, and school/work. Mean tie strength was 
closer to neutral than strong $(m=2.2$ on a scale of $1-3, S D=0.3)$, and ties provided 1.5 of three support types on average ( $S D=0.3)$, with emotionally supportive ties being most common ( $m=9.3, S D=5.7)$.

2.2.2. Interviewing. The interview protocol was developed for this study using the network map to guide the interview. Participants were given series of open-ended prompts, asked in varying order depending on their responses, and starting with a question about what stood out to them when they looked at their completed maps. The interview proceeded with questions about: individual network members, relationship strength, and support provided; the support types received from different domains, individuals who provided more support than others, and which types of support they had enough of or needed more of; member interconnection and how people knew each other; and any identified role models or informal mentors they'd included on the map. Next, participants were asked what they liked about their network and what they wished were different. Towards the end, participants were asked to identify potential obstacles to having their ideal network map, and/or suggested services to facilitate their ideal network map. The interview ended with any final thoughts for DHS or anything else they wanted to share about their support network. Combined, the network mapping and interview ranged in length from 45 minutes to two hours, with the average length of about 60 minutes.

\subsection{Analysis}

Interviews were recorded and professionally transcribed for analysis. One interview was with a Spanish-speaking youth and their bi-lingual ILP provider, who served as interpreter; the recording was transcribed in both languages, and the Spanish text was translated to English for the purposes of coding. Two project interns verified the transcripts, correcting errors while listening to the recorded interviews. The transcripts were uploaded into ATLAS.ti 7 software for deductive coding and analysis. Three coders coded one interview each to refine the initial codebook, which was primarily based on the protocol. Next, the first and second authors primary-coded two interviews each, then secondary-coded each other's interviews, meeting to discuss commonalities and discrepancies, and refining the codebook for 
inter-rater reliability. Coders repeated this process until each transcript was primary- and secondarycoded by the same two coders and both agreed on all assigned codes and excerpts in the entire dataset.

Next, the two authors conducted theoretical thematic analysis, which is a deductive method driven by research questions that approach the data from a particular theoretical perspective or conceptual framework (Braun \& Clarke, 2006). In this study, interviews were initially coded for descriptions of network characteristics and functionality (e.g., size, composition, interconnection, provision of multidimensional support, member stability) that are theorized to reflect support network capacity and overall stability. These concepts were embedded in the network map and the semistructured interview protocol, which significantly guided the initial deductive coding (e.g., participant references to the number of people in their networks were coded as network size, which is theoretically related to support network capacity). Initial coding also identified formal and informal social roles (e.g., foster parent, caseworker, siblings, mentors), as well as support types provided (e.g., emotional, concrete, multidimensional) and relationship characteristics (e.g., duration, conflict, closeness).

The interview also solicited perspectives on factors influencing whether participants received support by network members or overall (e.g., You mentioned that you feel more supported by your new foster parents. What are some of the ways they make you feel supported?), and the interviewer probed for recommendations around programs or services that they did (or potentially would) perceive as helpful in enhancing the support available through their networks (e.g., Did anyone help you stay in touch with your grandma? Is there anything DHS could do to help you connect with supportive peers?). The second stage of coding identified descriptions of barriers and facilitators to receiving support. These were analyzed as being thematically associated with network characteristics relating to functionality (e.g., difficulty making friends is a barrier that limits network size and therefore support capacity, where long-term relationships with relatives reflect network stability), as well as any inductive patterns that emerged during analysis (e.g., youth-driven services strengthen ties with providers and thus increase 
stability). The resulting themes summarize the facilitators, barriers, and recommended strategies, as organized within broader patterns of network functionality related to stability and support capacity.

\section{RESULTS}

Theoretical thematic analysis was used to identify salient themes in the described facilitators and barriers to participants receiving support, and to organize these using the broader concepts about support network functionality that provide the framework for the study. The identified themes related to support network capacity generally reflected interpersonal barriers that limited network size and the representation of particular social roles or support types in youth networks. The identified themes reflecting aspects of network stability were associated with the presence of a cohesive core network of strong and stable relationships facilitating individualized, multidimensional support. Lastly, we summarize emergent patterns of overall support network functionality, and report the most common youth-recommended strategies for support enhancement that were identified in the interviews. ${ }^{1}$

\footnotetext{
${ }^{1}$ Note that we refer to youth, young people, respondents, and participants interchangeably. Additionally, our findings reference "the system" in terms of the child welfare agency and related services (e.g., counseling), reflecting how this phrase was colloquially used by the participants.
} 
Table 1. Identified themes related to the theorized concepts and emergent enhancement strategies.

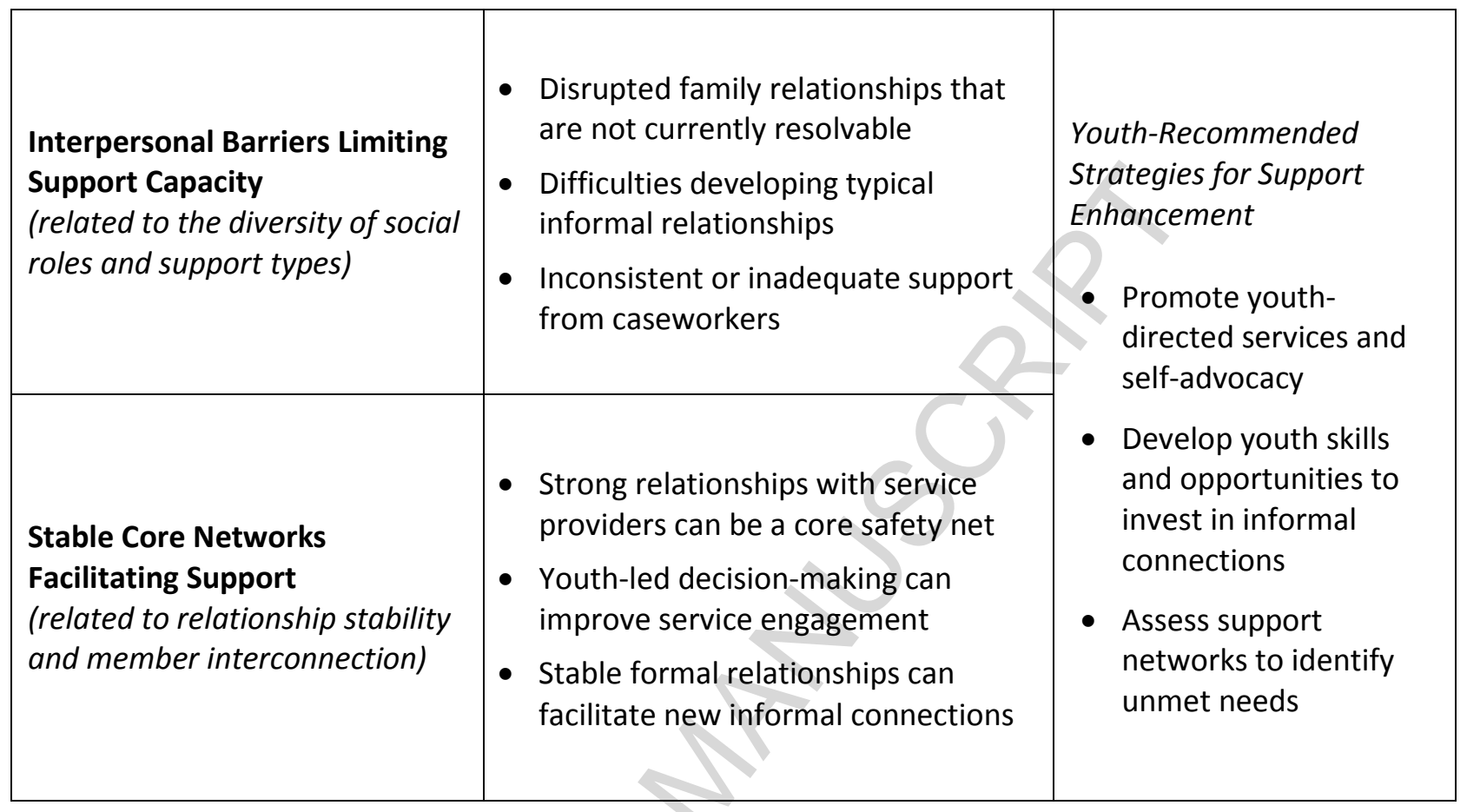

\subsection{Interpersonal barriers limiting support capacity}

Themes related to the capacity of support networks to adequately meet the needs of participants reflected interpersonal factors that would likely limit both the number and composition of support network members. These include youth descriptions of challenging relationships with formal service providers, and well as a range of participant difficulties in developing informal relationships with peers and supportive adults, and reports of unresolved conflicts with members of families of origin. Each of these barriers to the presence of typical relationships or specific types of support would impact the overall support network capacity.

3.1.1. Disrupted family relationships. Many youth reported having stable and supportive relationships with their family members-including parents, siblings, and other relatives - which would reflect typical support network capacity. However, many participants also reported disrupted relationships with members of their families of origin which limited the capacity of their support 
networks. These were generally attributed to circumstance, including relatives living far away or being incarcerated, or youth being in secure placement facilities or having court restrictions around contact. However, over a third of the sample described such disruption as being due to a family member's decision and/or lack of effort, where youth described feeling "dropped" or "disowned" by family members (in four cases, participants said this was because they had reported the abuse that led to their own foster placement). Lastly, six young people cited their own decision that they did not want contact with parents or relatives due to mental health challenges, substance abuse, or family history. Among those who described having disrupted ties with family, they consistently expressed that caseworkers had made efforts to facilitate contact or to resolve conflict, but there was nothing more the agency could do, with one youth stating, "With family issues and stuff, that's just between our family...nothing they can do about it." Notably, a few participants specifically described being hopeful about reestablishing or improving upon sibling relationships at some point in the future.

3.1.2. Difficulties developing informal relationships. When asked about typical natural support ties outside of family and service providers, such as peers and community members, over half of the sample described a degree of difficulty opening up, sharing their story, or connecting with new people. These challenges were generally attributed to aspects of their foster care experiences that shaped how they interacted with others, including learning to depend on themselves, feared stigma about their personal history, and a lack of opportunities to develop informal relationships. For example, multiple youth described developing a strong sense of self-reliance while in foster care:

You learn to distance yourself from people, and you just don't reach out to people as much because you're kind of taught to be self-sufficient, and that everybody kind of just leaves you. . . Like, people aren't willing to extend certain leniencies, or lend you things, or they're not willing to help you, because there's a stigma around being in foster care, that you're like a troubled kid, and so you kind of learn to do everything on your own.

Another young man addressed self-reliance by saying, "I just kind of, like, held my own hand" while another described his network as "a very tight circle, because you're least likely to get let down." 
Multiple young people described difficulty investing in relationships, often with self-awareness around this tendency ("if I was more open, I'd have a lot more people").

Many participants identified specific challenges around developing informal ties while they are in foster care, with one young person saying the following:

I went into foster care at eighth grade, so I wasn't super-young, but I still found myself losing a lot of what I thought was support networks, like, the first couple of years that I was in foster care, because I moved around a lot, and so it was hard to make any lasting connection with anybody.

Some spoke specifically about difficulty making friends, or not even wanting to have friends, due to their personal history. One young woman described learning to push past a tendency to be closed off:

It was really hard for me to make friends. I still don't have a lot of friends, but, like, you have to open up [laughs]. Because, like, I don't want to get made fun of for my story and what's happened to me and stuff.

Youth in residential programs were most likely to discuss the impact of these placements (or stigma around the reasons they were placed there) on typical peer relationships, including the circumstantial nature of contact with program peers, difficulty maintaining ties with old friends, and a lack of opportunities to develop new ties outside of the placement. When asked about his ideal network, one youth in long-term treatment said that he'd have more friends ("I like having people knowing me as I am, and not why I'm here ") and described his worry that being away would change how his old friends viewed him. Another young woman in a residential program said she wanted more friends on her network map, "but it just kinda sucks not having anyone to talk to besides people here...I really wouldn't call them friends... they have their own problems going on." Most youth in supervised group settings observed that rules around when and how they can contact people or spend time in the community restricted their ability to experience typical relationships. For these youth, what was often lacking in their networks were self-selected supports, described by one young man as "people I choose to have a relationship with, not ones I'm forced to have a relationship with, because of circumstance." 
3.1.3. Inadequate caseworker support. Participants were asked about the service-providing relationships on their maps, as well as those that were absent (e.g., if they did not identify a current caseworker as supportive). Analysis identified a subgroup of eight participants who lacked support from child welfare agency caseworkers specifically, resulting in participant confusion about case status, service access, and placement options. For example, multiple youth identified significant difficulty initiating communication with caseworkers, despite repeated attempts ("I've been trying and trying and trying"). One young man described his caseworker as only showing up before scheduled court hearings, but otherwise not responding to messages from the youth: "I know he's busy, but he could honestly get back to me, let me know something." Multiple youth similarly acknowledged that their caseworker had a full caseload, but expressed frustration around their inability to get timely support, and described how this negatively impacts the relationship: "They have busy schedules, we get that, but at least meet with these kids, not even once, like twice a month. My people need to be focused, because I need them." A few mentioned confusion about who their service providers were, as this could change unexpectedly. One young man explained:

I used to have a different caseworker, since I first been in foster care, since I was like eight years old. And we just switched ... I don't know what happened, like, they didn't even tell us that, they just like "Oh how you feel about getting a new caseworker?" and that was that...I don't know what happened to her.

One young woman was told that she had an assigned independent living program worker, but the service was inaccessible from her perspective: “I've never met them. My caseworker's like, "Oh, but you have one", but I don't even know who they are." Another summarized his discouraging experiences with multiple caseworkers, saying, "They've been significant, even though they're not helpful. They're all the same amount of...I mean, one was nicer than the others, but they're so overworked." Overall, multiple youth described a poor relationship with a caseworker as limiting the capacity of their support network, both by being a barrier to direct support and limiting their indirect access to other services and support.

\subsection{Stable core networks facilitating support}


On the other hand, many participants described network stability as facilitating support through positive, enduring, and interconnected formal and informal relationships. Identified themes include descriptions of supportive relationships with a "team" of service providers, youth having a voice in decision-making, and the intentional facilitation of typical peer and community connections. The presence of a core network of such relationships is likely to provide stable and multidimensional support individualized to the youth's preferences and needs, much as a typical family is expected to provide during adolescence. Overall, a large subgroup described service providers (including paid caregivers) as stable network members, in some cases in the absence of longer-term informal ties, including family.

3.2.1. Service providers as a core safety net. Nine youth referenced having generally supportive service-providing relationships - either with individuals or with an interconnected support "team" - and many identified aspects of reciprocity typically found in normative positive relationships between youth and adults. Youth referenced patterns of negotiation and compromise, listening and understanding of each other's perspectives, and providers approaching decision-making with an open mind and sense of humor. One young woman said her "support system" wants the best for her, and another described a similar sense of enveloping support, where if she were to ever struggle, their response would be "you're not going anywhere, you have a stable environment, you have us." Long-time caseworkers played a particularly important role that was often attributed to the longevity of the relationship, with one participant saying, "She knows everything...she knows what I want and what I need and she knows me as a person." Another young woman felt that her experience of caseworkers "really going above and beyond" was unusual compared to other foster youth:

It's been that way for all the caseworkers I've had, they've been really proactive and really interested in what I'm interested in, and interested in supporting me, and they really care about what I want to do and they want to be a part of it.

Multiple youth referenced feeling that supporting them was "not just a job", a few mentioned specific providers who "genuinely care" about them, and a few described providers as a trusted authority figure 
or like a parent. Multiple youth described long-time caseworkers as having watched them grow up, consistently being there through positive and negative experiences over the years.

On the other hand, a few youth described positive relationships with multiple providers, but were aware they lack longer-term ties providing informal support, particularly with family. One young woman explained, "where I feel like more people would have like a heavier amount of people over here by family, mine's like paid positions, which is like a normal foster kid thing." Others expressed awareness that paid relationships will not be long term, with one describing, "they're all just gonna fade away, just because of confidentiality and keeping their lives separate." Another shared fears around relying on providers as her "biggest support" as she approaches the end of her eligibility for foster care services: "They should be providing more services that are long-term supports. Because all these people, bestcase scenario, I keep them as long as I can, they're gone at 21."

3.2.2. Youth-led service engagement. When asked about factors that facilitated receiving support from service providers, multiple youth described a time when they had voiced a preferencefor example, in choosing their placement type, or choosing between two treatment programs-and felt they were heard in a way that influenced the decision. A few participants described learning to balance a sense self-reliance with growing self-advocacy within the system. For example, multiple youth described a turning point where they decided to advocate for themselves and "work with the system" to improve their situation, with one young man in residential treatment telling his service team, "I'm sorta getting tired of you guys governing my life. I'm stepping up to the plate and saying this is how it's gonna happen." When asked what advice they would give to other foster youth, multiple participants encouraged others to take advantage of the support and resources available through the child welfare system while they could. As one young man described, "Even though I don't like DHS, I still have to work with them to get what I want." Some youth described how it helped for them to learn over time what services and supports were available, and what service providers can and cannot do, as one young 
woman described: "I used to think [my caseworker] would do whatever he wants. . but now I realize

DHS only allows him to do so much, so I appreciate more what he does for me." One young woman who felt supported by her team expressed a similar sense of reciprocity:

If you have concerns and stuff, or complaints, take a look at what you're doing to see if you can help fix it before you blame it all on them, you know? There is stuff that's wrong with the system . . . but I feel that it's not all the system's fault. . . . some people need to work on their ability to connect with others, even though that may be really super-hard.

As seen in these quotes, youth-directed services and supports were often described as facilitating youth engagement and strengthening collaborative and reciprocal relationships with providers.

3.2.3. Formal relationships can facilitate informal connections. Participants also identified facilitators to developing supportive relationships with peers and community members. As reported above, about half of all participants described difficulty opening up or connecting with people. Among those who described these experiences as being in the past, some attribute the change to placement stability allowing them to be able to invest in relationships. One young person described moving to a stable and supportive foster home, where she was then able to develop close friendships because she was "in a carefree environment ... so I could focus on relationship building." Another expressed a similar sentiment about making an effort to "reinvent" herself with new peers at a new school, after she moved to a stable placement with relative foster parents. Another young woman described having an understanding foster parent who facilitated developmentally-typical experiences:

She gives us a lot of slack, you know? But not the kind of slack that lets us go off and do stuff that's bad, but the kind of slack to be kind of a normal teenager. She's just kind of trusting me to choose the right people to hang out with, you know?

Similarly, a few appreciated caregivers who gave them space to manage typical adolescent romantic problems without becoming overprotective, "just because we have a rough patch or a bad moment."

Youth who reported feeling connected to various communities described participation opportunities such as youth advisory boards, church groups, school sports and clubs, and recreational 
activities. Some gave credit to foster parents and caseworkers for supporting and facilitating such informal relationship-building based on the young person's interests. Others advocated for the child welfare system to provide more developmentally-appropriate opportunities for low-barrier social participation, such as basketball tournaments or holiday parties with same-age peers in foster care, to facilitate new connections and potential friendships. When asked what advice they would give to other young people around making new connections, multiple youth recommended taking advantage of available participation opportunities. Some specifically described advocating for themselves to participate in their chosen community activities, with one young woman recommending that service providers prioritize youth access to such opportunities, where youth are "getting to know people in the community so that you can build some lasting relationships around something like that."

A few participants described similar individualized support from caregivers and service provider teams as facilitating the development of typical informal relationships with peers and community groups.

\subsection{Emergent patterns of support network functionality}

Overall, about half of the sample described having a functional support network with adequate stability and capacity to address youth needs. These support systems included core networks of supportive, strong, and interconnected ties, usually anchored by longer-term placement in a familybased setting, where many also had regular support from their family of origin. Youth with such networks tended to report longer-term, positive relationships with caseworkers and service providers, and foster parents that offered multi-dimensional support; in some cases, participants specifically described caseworkers as parents or referred to their foster caregivers as "mom" and/or "dad". Additionally, these networks included ties to peers and informational supports (e.g., teachers), as well as ties between formal providers and informal network members (e.g., an out-of-state relative calling in to service team meetings). Overall, these networks seem to provide normative developmental support, 
such as celebrating youth progress, encouraging interests, and attending youth events, in addition to providing a reliable safety net when youth struggled with developmentally-typical needs.

For many of these youth, the experience of mapping and discussing their networks underscored that they had a range of stable and supportive relationships and validated a perception that that their support needs were generally being met. For example, one participant described adequate support by saying, "I have strong connections between friends, family, and community, and I have a lot of emotional support." Another stated: "I like that there's a lot of connections and that almost everybody can give me informational support, and there's a whole lot of emotional support from almost everybody." Many who said they were satisfied with the support they got from such networks had difficulty identifying potential areas for their own network enhancement, and some stated that no additional efforts were needed (e.g., "I don't need or want any more help. I'm perfect where I'm at.")

In comparison, about half of the participants reported having less robust support networks with identified limitations in support capacity and network stability. These were generally smaller, less interconnected networks, with no core set of longer-term ties (e.g., "I really struggled to come up with some people to put on here"). These youth were less likely to be living in a family-based foster home or with relatives, and they more often reported not living in an approved placement or being in a group placement at the time of the interview (including residential treatment, semi-secure group homes, and a homeless shelter). These youth also reported a lack of long-term and/or positive relationships with caseworkers and other service providers, and described negative experiences of provider turnover. A few were specifically frustrated with unresponsive caseworkers, and voiced a need for more robust concrete and informational support. Youth with such networks were most likely to mention a lack of self-selected friends and community supports due to foster care factors (e.g., placement instability, limited opportunities in restricted placements, lack of trust or "openness"). Additionally, a small subgroup had strong relationships with service providers, but lacked informal ties for long-term support. 
For many, the network mapping process introduced language to describe their networks in terms of support capacity, and a few said they wished they had done this at an earlier stage. For about a third of the sample, the process helped to identify that they were feeling disconnected from support systems and were struggling with unmet needs. For this subgroup, network mapping helped identify individual people, categories of network members, or kinds of relationships that were missing from the youth's perspective. One participant described his ideal network by summarizing, "I wish my older brother was a strong line. I wish I had nothing but strong lines, and I wish I had more names." Many youth wanted more frequent caseworker support ("he needs to step it up a little bit, because I need help"), while some noticed that they had named providers as primary supports in the absence of familybased ties ("the consistency is mostly in the paid people"). For some, it gave perspective on how weaker or absent relationships may be impacting them. For example, when asked what service providers could do to better support her, one youth pointed to the family section of her map and said, "Understand that I needed this and I don't have it, and that is going to affect the way that I work with them."

\subsection{Youth-recommended strategies for support network enhancement}

Participants were specifically asked what the child welfare system could do more of (or do differently) to help resolve identified barriers to receiving formal and informal support. Those who lacked a core support network, were disconnected from services, or were reliant on paid providers, were more likely to identify their own need for support network enhancement, especially around improving collaboration with providers and developing supportive peer relationships and community connections. However, some expressed awareness of entrenched challenges, for example, that caseworkers are often overloaded and have high job turnover; participants did not identify related strategies to overcome such systemic barriers, but rather shared the challenge of their individual circumstances. There were also no new strategies to resolve disrupted family relationships identified in this study. Rather, this was an interpersonal challenge experienced by some youth with more and less 
robust networks overall, and some appreciated that this was a barrier that service providers had tried to address. When prompted to suggest new strategies to resolve disrupted family ties, a few participants identified family counseling or skills training as potential approaches to improve these relationships.

On the other hand, many of the participants who described presently feeling supported by their networks also reflected on how they had overcome past challenges (opening up to new people, for example, or working with providers) and offered advice to other young people in care. The following are the most salient youth-recommended support enhancement strategies emerging from the analysis:

- Promote youth-led decision-making and self-advocacy around desired services and supports

- Develop youth skills and opportunities to invest in supportive informal connections with peers, adults, and community groups

- Use network assessment tools to better understand youth support network capacity and to identify and address individual unmet needs

We discuss these strategies further below, in the context of existing intervention approaches.

\section{DISCUSSION}

The aims of this study were to explore support network functionality among a diverse sample of transition-age foster youth, and to identify any related support network enhancement strategies. For this analysis, support was conceptualized as a function of both a network's capacity to provide adequate support to address young adult needs, and of network stability, which reflects cohesion within and across relationships to facilitate multifaceted support over time. Overall, our findings confirm the presence of emergent patterns of support network functionality which reflect the impact of foster care factors on indicators like composition and interconnectedness. We discuss these patterns below, and then explore youth-recommended strategies for support network enhancement, which focused on youth-directed service and access to informal relationship development skills and opportunities.

\subsection{Support network functionality}


In this sample, there were patterns of overall network functionality that confirm much of what we know about how foster care factors can influence socioemotional development, the availability of parent figures and informal support resources, and ongoing service engagement as young people age out of the child welfare system (e.g., Cushing, Samuels, \& Kerman, 2014; Singer, Cozner, \& Berzin, 2013; Zinn, Palmer, \& Nam, 2017). Specifically, the primary barriers identified here related to challenges that impacted the presence or absence of specific types of relationships and/or support, which limits both the size and diversity of the networks, which are key factors related to network capacity to provide a range of support types as needed to address youth needs (Blakeslee, 2015; Wellman \& Wortley, 1990). Additionally, many youth described networks limited by disrupted family connections that were not currently resolvable from the youth perspective, inconsistent relationships with caseworkers that contributed to an overall feeling of disconnection from formal transition services and supports, and multi-level factors potentially limiting youth openness to relationship development and help-seeking (similar to findings reported in Pryce, Napolitano, \& Samuels, 2017).

Similarly, the concept of overall network stability reflects how longer-term and interconnected relationships provide multidimensional and individualized support over time. Here, there was a clear subgroup of young people describing a "support system" anchored by long-term relationships, including service providers and foster caregivers, many of whom were relatives or kin. This recalls previous descriptions of youth in care experiencing "felt security" (Cashmore \& Paxman, 2006), and echoes person-oriented research that consistently finds a large subgroup of foster youth who are doing relatively well and who also report high social support and positive relationships with providers (e.g., Keller et al., 2007). Relatedly, the most salient facilitators identified in this analysis were associated with "family-like" functions of network, and did not reflect aspects of network size or the presence of specific kinds of people or support types (as referenced as being lacking in the identified barriers). This may also explain patterns in youth-recommended strategies for network enhancement, which were less likely to 
focus on specific relationships or support types, and more likely to be skill development strategies to strengthen youth capacity to access potential formal and informal support resources in general.

\subsection{Strategies for support network enhancement}

We solicited youth perspectives on how child welfare systems could better resolve barriers to network capacity and stability in ways that would facilitate support. Some identified barriers were relatively consistent regardless of overall network functionality; for example, disrupted family relationships were experienced by some youth with and without robust support networks, as were difficulties making peer connections. However, the finding that youth did not identify family support as an area for new or additional services or programming was unexpected, and may reflect the impact of relatively recent policy shifts to prioritize family connections and relational permanency for older youth in care. As systems continue to focus on this domain, strategies may include increasing the availability of relational skill-building programming, ensuring access to youth and family counseling, and whenever possible, allowing older youth to determine which family members that want to have contact with. Similarly, there were few identified barriers or strategies related to foster parents, which may be because youth who felt well-supported by their network in this study were more likely to be in stable foster placements. Lastly, one of the most salient identified barriers to support provision related to difficult relationships with caseworkers specifically, which impacted the capacity of their networks by limiting direct and indirect access to concrete and informational support and resources. Participants who experienced this generally referenced awareness of workload, and did not identify potential strategies to improve these relationships, outside of the areas discussed below. Rather, youth-identified strategies focused on recognizing unmet support needs and promoting youth skills and opportunities to address these needs by directing their own engagement with formal and informal support resources.

4.2.1. Promoting youth-directed services. Self-determination was a consistent theme for youth who felt well-supported in relationships with service providers in their networks, as well as those who 
specifically described a turning point when they began to "work with the system" and could direct services according to their preferences and self-identified needs. Importantly, a perceived lack of selfdetermination was identified among youth who voiced unmet support needs, including frustrating difficulties understanding and accessing the services for which they were eligible, or not having voice and choice in important placement decisions. A few participants specifically recommended that youth learn more about the services available to them and begin advocating for themselves, although arguably this is not always feasible for youth who are having difficulty communicating with caseworkers around basic needs. We do know that consistent coaching to increase the application of self-determination skills can improve outcomes, for example by teaching youth how to lead their own transition planning meetings (e.g., Powers et al., 2012; Powers et al., 2018). However, skill-building strategies to facilitate supportive ties to service providers and other formal resources must account for youth readiness to invest in these relationships, given prior experiences influencing mental health and attachment (e.g., impacting post-secondary outcomes: Morton, 2018; Okpych \& Courtney, 2018).

4.2.2. Developing supportive informal connections. Many participants described having difficulty opening up, sharing their story, or connecting with people. Among those who overcame these challenges, some attributed the change to placement stability which allowed them to intentionally focus on relationships, or gaining an understanding that they needed to be more open, even when this was difficult due to past experiences. This recalls the tendency towards negative perceptions around helpseeking (Pryce, Napolitano, \& Samuels, 2017; Seita, Day, Carrellas, \& Pugh, 2016) associated with subgroups of young people aging out of care. Such limitations can potentially be addressed through a range of approaches that focus on building socio-relational skills and strengthening relationships with specific non-parental adults (e.g., Greeson \& Thompson, 2017; Hall \& Jones, 2018; Nesmith \& Christophersen, 2014; Spencer, Gowdy, Drew, \& Rhodes, 2018). 
However, young people in foster care also need to have adequate opportunities to invest in typical peer and community-based relationships, and a related recommendation would be to make these opportunities more available where they are lacking. Some youth said they would be interested in new kinds of activities to informally meet people with shared interests, including other youth in foster care, as this would address concerns about others understanding their circumstances. Further, youth who did feel supported in developing informal ties referenced participating in typical youth groups and activities, which has been recognized as a challenge in child welfare policy and practice; recent legislation promotes a sense of "normalcy" for youth in foster care (see Simmons-Horton, 2017, for related policy analysis) to support these young people in having typical teen social experiences.

Additionally, supporting new community ties may include first identifying activities or settings that youth are interested in and then facilitating their ongoing participation; supported experiential activities are a key component in evidence-based models to improve foster care outcomes (e.g., Phillips et al., 2015; Powers et al., 2018). Further, there is some evidence that shorter-term social participation interventions, primarily peer support groups and supported community activities, can improve social inclusion and interpersonal functioning among transition-age young people receiving mental health services (Webber \& Fendt-Newlin, 2017), which is also a promising approach for older youth in foster care. Importantly, a common thread in these approaches is a peer support and/or near-peer mentoring component, where young people with similar lived experience can normalize difficult circumstances and promote informal relationship-building and youth-directed service engagement. Additionally, foster youth advisory boards and related youth leadership development activities provide youth opportunities to work with similar peers, meet supportive adults, and share their lived experiences with child welfare system decision-makers to improve services (Forenza, 2016; Havlicek, Lin, \& Villapando, 2016).

4.2.3. Support network assessment. Facilitated support network mapping with follow-up discussion seems to be a feasible practice strategy to identify a subgroup of youth experiencing 
challenges related to prior network disruption, inhibited social development, and resulting limitations on the capacity and/or stability of their support networks to address their present and future needs. For example, service providers can assess whether there are stable family-based connections and parent figures in the network, and whether there are informal mentors and typical peer supports (as in Blakeslee, Kothari, McBeath, Sorenson, \& Bank, 2017). Such network assessment can generate potential areas of support enhancement required to increase transition readiness, whether this is to facilitate new connections to increase network diversity, engage youth in discussions about relationship-building skills or help-seeking attitudes, or connect young people to near-peers with similar lived experiences. Additionally, support network mapping offers potentially new language to assist youth in describing specific emotional, informational, and/or concrete needs that are not otherwise being recognized as contributing to transition-related difficulties that youth are experiencing. For example, network assessment may reveal conflict with important providers, disconnection from social domains, or a lack of informal supports. Such conversations may be especially important when youth are not in family-based placements or otherwise connected to a core network, not service-connected, and/or when services are soon to end.

\subsection{Implications for Research and Practice}

The preceding theoretical thematic analysis was undertaken to advance a potential framework for understanding how foster placement impacts support network structure and to inform ongoing research and practice efforts to address resulting limitations through services. In this sample, the important role of long-term service providers and foster caregivers in anchoring support networks with the capacity and stability to promote typical adolescent development was clear. This may reflect cases where services are providing the comprehensive support they are designed to facilitate for youth in outof-home placement, although youth reliance on these formal relationships as core network members must be balanced with provider awareness that many of these service-oriented relationships can be 
expected to weaken or end when youth age out of foster care. On the other hand, youth-identified strategies for support network enhancement tended to focus on facilitating opportunities for youth to have more voice and choice in service delivery, including the freedom and skills to develop supportive informal relationships which have the potential to outlast child welfare system involvement.

Importantly, the Administration for Children and Families (ACF) prioritizes a preventative focus on social and emotional well-being to allay the poor outcomes experienced by former foster youth (HHS, 2012), and recognizes the role of universal Independent Living Programs (ILP) in these efforts. Federallysupported ILPs are a prevalent service mechanism available to foster youth, and have historically been charged with increasing education, employment, and self-sufficiency skills, but recent federal-level planning also prioritizes "soft" developmental assets like social connections and psychosocial skills (Courtney et al, 2017; McDaniel et al., 2014). ILP settings may be a suitable context for implementing the support enhancement strategies presented here, which focus on self-determination and socio-relational skill development, and facilitation of opportunities to access informal peer support and communitybased connections. Regardless of service setting, however, such an approach can be grounded in support network assessment in practice, similar to the protocol used here for research purposes.

\subsection{Limitations}

While study participants were diverse in terms of race/ethnicity, LGBTQ identities, placement types, and urbanicity, the findings reflect the experiences of a purposive sample that was largely recruited through service providers who were in a position to act as gatekeepers in determining which youth heard about the study. Additionally, a portion of the sample was recruited by convenience at a state-sponsored foster youth event. Despite regional variation within the state of Oregon, this sample is not representative of a larger statewide or national population, and more likely reflects the potentially more positive experiences of a group of relatively service-connected young people, with additional oversampling of LGBTQ-identified youth. Given these considerations, our exploratory findings reflect 
themes emerging from interviews with a group of young people who are more likely to have servicerelated needs being met, and the experiences of a group of less service-connected youth overall may have resulted in much different themes and recommendations around support network enhancement.

Second, the theorized network concepts underlying the mapping and interview protocol, including language for identifying strength of ties and types of support, were prescriptive; this limited the breadth or depth of the findings in terms of our ability to reflect unique experiences or perspectives in the participants' own words. Similarly, the deductive analytic approach was specifically designed to identify facilitators and barriers to receiving support as these were related to the predetermined concepts of interest, and was conducted to apply this framework in ways that inform existing practice, as well as potential new approaches for addressing support limitations. Other analyses of these data will explore participants' supportive relationships with less prescriptive aims to allow for more inductive findings, including forthcoming narrative analysis of network disruption. Lastly, we did not conduct mixed method or quantitative analysis of the support network map indicators or participant demographics for this study, which can be further explored in future studies, including analysis by age, gender, LGBTQ identification, race/ethnicity, as well as by urbanicity and placement type and duration.

\subsection{Conclusion}

Health and well-being can be profoundly influenced by social networks, and addressing social isolation and strengthening ties between people is considered a "grand challenge" in social work (Lubben, et al., 2015). Among youth exiting foster care, we know that many have relatively sparse social networks with limited access to support and resources, and many have developed hard-earned skepticism about seeking support through formal services and/or informal relationships. In addition to the ongoing implementation of best practices to promote both legal and relational permanency for youth in care, we can continue to improve our understanding about how to enhance the capacity of these support networks when they have been structurally impacted by the experiences that led to, and 
result from, child welfare system involvement. This study confirms our understanding that many young people in foster care (and nearly half of the youth in this sample), do feel well-supported by a family-like core network providing consistent and individualized formal and informal support. On the other hand, many of the youth interviewed here identified unmet support needs that reflected a limited range of supportive connections and the lack of a stable support network, which suggests ongoing research and intervention development around systematically assessing and addressing these network limitations. This study also highlights the perspective of young people aging out of foster care on potential areas for enhancing support through services and programming, which can focus on factors that can limit the capacity of youth support networks by inhibiting informal relationship development, and acknowledge the pivotal role of service providers and foster caregivers in facilitating long-term network stability through individualized, youth-led approaches. 
The authors are not aware of any conflicts of interest related to this study.

\section{Funding acknowledgement:}

Preparation of this article was supported in part by funding from the National Institutes of Health Award Number UL1GM118964, administered by the National Institute of General Medical Sciences. The content is solely the responsibility of the authors and does not necessarily represent the official views of the National Institutes of Health. 


\section{References}

Ahrens, K. R., DuBois, D. L., Garrison, M., Spencer, R., Richardson, L. P., \& Lozano, P. (2011). Qualitative exploration of relationships with important non-parental adults in the lives of youth in foster care. Children and Youth Services Review, 33, 1012-1023.

Avery, R. J. (2010). An examination of theory and promising practice for achieving permanency for teens before they age out of foster care. Children and Youth Services Review, 32, 399-408.

Berzin, S. C., Singer, E., \& Hokanson, K. (2014). Emerging versus emancipating: the transition to adulthood for youth in foster care. Journal of Adolescent Research, 29(5), 616-638.

Biglan, A., Flay, B. R., Embry, D. D., \& Sandler, I. N. (2012). The critical role of nurturing environments for promoting human well-being. American Psychologist, 67(4), 257-271.

Blakeslee, J. E. (2012). Expanding the scope of research with transition-age foster youth: applications of the social network perspective. Child \& Family Social Work, 17(3), 326-336.

Blakeslee, J. E. (2015). Measuring the support networks of transition-age foster youth: Preliminary validation of a social network assessment for research and practice. Children and Youth Services Review, 52, 123-134.

Blakeslee, J. E., \& Keller, T. E. (2016). Assessing support network stability with transition-age foster youth: Measuring change over time. Research on Social Work Practice, DOI: 1049731516678662.

Blakeslee, J., Kothari, B. H., McBeath, B., Sorenson, P., \& Bank, L. (2017). Network indicators of the social ecology of adolescents in relative and non-relative Foster households. Children and Youth Services Review, 73, 173-181.

Braun, V., \& Clarke, V. (2006). Using thematic analysis in psychology. Qualitative Research in Psychology, 3(2), 77-101. 
Brooks, F. M., Magnusson, J., Spencer, N., \& Morgan, A. (2012). Adolescent multiple risk behaviour: an asset approach to the role of family, school and community. Journal of Public Health, 34(S), i48¡56.

Brown, A., Courtney, M. E., \& McMillen, J. C. (2015). Behavioral health needs and service use among those who've aged-out of foster care. Children and Youth Services Review, 58, 163-169.

Cashmore, J., \& Paxman, M. (2006). Predicting after-care outcomes: the importance of 'felt' security. Child \& Family Social Work, 11(3), 232-241.

Coleman, J. S. (1988). Social capital in the creation of human capital. American Journal of Sociology, 94, S95-S120.

Courtney, M. E., Okpych, N. J., Park, K., Harty, J., Feng, H., Torres-Garcia, A., \& Sayed, S. (2018). Findings from the California Youth Transitions to Adulthood Study (CalYOUTH): Conditions of youth at age 21. Chicago, IL: Chapin Hall at the University of Chicago.

Courtney, M. E., Pergamit, M., McDaniel, M., McDonald, E., Giesen, L., Okpych, N. \& Zinn, A. (2017). Planning a Next-Generation Evaluation Agenda for the John H. Chafee Foster Care Independence Program, OPRE Report \#2017-96, Washington, DC: Office of Planning, Research, and Evaluation, Administration for Children and Families, U.S. Department of Health and Human Services.

Cushing, G., Samuels, G. M., \& Kerman, B. (2014). Profiles of relational permanence at 22: Variability in parental supports and outcomes among young adults with foster care histories. Children and Youth Services Review, 39, 73-83.

Daining, C., \& DePanfilis, D. (2007). Resilience of youth in transition from out-of-home care to adulthood. Children and Youth Services Review, 29, 1158-1178.

Dworsky, A., Napolitano, L., \& Courtney, M. (2013). Homelessness during the transition from foster care to adulthood. American Journal of Public Health, 103(S2), S318-S323. 
Forenza, B. (2016). Opportunity role structure, social support, and leadership: Processes of foster youth advisory board participation. Journal of Community Psychology, 44(7), 904-918.

Geenen, S., \& Powers, L. E. (2007). "Tomorrow is another problem": The experiences of youth in foster care during their transition into adulthood. Children and Youth Services Review, 29, 1085-1101.

Goodkind, S., Schelbe, L. A., \& Shook, J. J. (2011). Why youth leave care: Understandings of adulthood and transition successes and challenges among youth aging out of child welfare. Children and Youth Services Review, 33, 1039-1048.

Greeson, J. K., \& Bowen, N. K. (2008). "She holds my hand" The experiences of foster youth with their natural mentors. Children and Youth Services Review, 30, 1178-1188.

Greeson, J. K., \& Thompson, A. E. (2017). Development, feasibility, and piloting of a novel natural mentoring intervention for older youth in foster care. Journal of Social Service Research, 43(2), 205-222.

Gypen, L., Vanderfaeillie, J., De Maeyer, S., Belenger, L., \& Van Holen, F. (2017). Outcomes of children who grew up in foster care: Systematic-review. Children and Youth Services Review, 76, 74-83.

Hall, S. F., \& Jones, A. S. (2018). Implementation of intensive permanence services: a trauma-informed approach to preparing foster youth for supportive relationships. Child and Adolescent Social Work Journal, 1-12.

Havlicek, J. R., Garcia, A. R., \& Smith, D. C. (2013). Mental health and substance use disorders among foster youth transitioning to adulthood: Past research and future directions. Children and Youth Services Review, 35, 194-203.

Havlicek, J., Lin, C. H., \& Villalpando, F. (2016). Web survey of foster youth advisory boards in the United States. Children and Youth Services Review, 60, 109-118. 
IOM (Institute of Medicine) and NRC (National Research Council). 2013. Improving the health, safety, and well-being of young adults: Workshop summary. Washington, DC: The National Academies Press.

James, S., Landsverk, J., \& Slymen, D. J. (2004). Placement movement in out-of-home care: Patterns and predictors. Children and Youth Services Review, 26, 185.

Jones, L. (2013). The family and social networks of recently discharged foster youth. Journal of Family Social Work, 16(3), 225-242.

Keller, T. E., Cusick, G., \& Courtney, M. E. (2007). Approaching the transition to adulthood: Distinctive profiles of adolescents aging out of the child welfare system. Social Service Review, 81(3), 453484.

Lubben, J., Gironda, M., Sabbath, E., Kong, J., \& Johnson, C. (2015). Social isolation presents a grand challenge for social work (Working Paper No. 7). Grand Challenges for Social Work Initiative: American Academy of Social Work and Social Welfare.

McCoy, H., McMillen, J., \& Spitznagel, E. (2008). Older youth leaving the foster care system: Who, what, when, where, and why? Children and Youth Services Review, 30, 735-745.

McDaniel, M., Courtney, M. E., Pergamit, M. R., \& Lowenstein, C. (2014). Preparing for a "Next Generation" Evaluation of Independent Living Programs for Youth in Foster Care: Project Overview, OPRE Report \# 2014-71. Washington, DC: Office of Planning, Research and Evaluation, Administration for Children and Families, US Department of Health and Human Services.

McPherson, K. E., Kerr, S., Morgan, A., McGee, E., Cheater, F. M., McLean, J., \& Egan, J. (2013). The association between family and community social capital and health risk behaviours in young people: an integrative review. BMC Public Health, 13(1), 971.

Moody, J., \& White, D. R. (2003). Structural cohesion and embeddedness: A hierarchical concept of social groups. American Sociological Review, 68, 103-127. 
Morton, B. M. (2018). The grip of trauma: How trauma disrupts the academic aspirations of foster youth. Child Abuse \& Neglect, 75, 73-81.

Munson, M. R., Lee, B. R., Miller, D., Cole, A., \& Nedelcu, C. (2013). Emerging adulthood among former system youth: The ideal versus the real. Children and Youth Services Review, 35, 923-929.

Munson, M. R., \& McMillen, J. C. (2009). Natural mentoring and psychosocial outcomes among older youth transitioning from foster care. Children and Youth Services Review, 31, 104-111.

Nesmith, A., \& Christophersen, K. (2014). Smoothing the transition to adulthood: Creating ongoing supportive relationships among foster youth. Children and Youth Services Review, 37, 1-8.

Okpych, N. J. (2015). Receipt of independent living services among older youth in foster care: An analysis of national data from the US. Children and Youth Services Review, 51, 74-86.

Okpych, N. J., \& Courtney, M. E. (2018). The role of avoidant attachment on college persistence and completion among youth in foster care. Children and Youth Services Review, 90, 106-117.

Paulsen, V., \& Berg, B. (2016). Social support and interdependency in transition to adulthood from child welfare services. Children and Youth Services Review, 68, 125-131.

Perry, B. (2006). Understanding social network disruption: The case of youth in foster care. Social Problems, 53(3), 371-391.

Phillips, L. A., Powers, L. E., Geenen, S., Schmidt, J., Winges-Yanez, N., McNeely, I. C., ... \& Bodner, C. (2015). Better Futures: A validated model for increasing postsecondary preparation and participation of youth in foster care with mental health challenges. Children and Youth Services Review, 57, 50-59.

Powers, L. E., Fullerton, A., Schmidt, J., Geenen, S., Oberweiser-Kennedy, M., Dohn, J. Et al. (2018). Perspectives of youth in foster care on essential ingredients for promoting self-determination and successful transition to adult life: My life model. Children and Youth Services Review, 86, 277-286. 
Powers, L. E., Geenen, S., Powers, J., Pommier-Satya, S., Turner, A., Dalton, L. D., ... \& Swank, P. (2012). My Life: Effects of a longitudinal, randomized study of self-determination enhancement on the transition outcomes of youth in foster care and special education. Children and Youth Services Review, 34, 2179-2187.

Rutman, D., \& Hubberstey, C. (2016). Is anybody there? Informal supports accessed and sought by youth from foster care. Children and Youth Services Review, 63, 21-27.

Samuels, G. M., \& Pryce, J. M. (2008). "What doesn't kill you makes you stronger": Survivalist selfreliance as resilience and risk among young adults aging out of foster care. Children and Youth Services Review, 30, 1198-1210.

Shpiegel, S., \& Ocasio, K. (2015). Functioning patterns among older adolescents in foster care: Results from a cluster analysis. Children and Youth Services Review, 58, 227-235.

Seita, J., Day, A., Carrellas, A., \& Pugh, G. L. (2016). Assessing the help-seeking behaviors of foster care alumni within their own social networks. Journal of Sociology, 4(2), 1-10.

Shook, J., Goodkind, S., Pohlig, R. T., Schelbe, L., Herring, D., \& Kim, K. H. (2011). Patterns of mental health, substance abuse, and justice system involvement among youth aging out of child welfare. American Journal of Orthopsychiatry, 81(3), 420-432.

Simmons-Horton, S. Y. (2017). Providing age-appropriate activities for youth in foster care: Policy implementation process in three states. Children and Youth Services Review, 82, 383-391.

Singer, E. R., Berzin, S. C., \& Hokanson, K. (2013). Voices of former foster youth: Supportive relationships in the transition to adulthood. Children and Youth Services Review, 35, 2110-2117.

Spencer, R., Gowdy, G., Drew, A. L., \& Rhodes, J. E. (2018). “Who knows me the best and can encourage me the most?": Matching and early relationship development in youth-initiated mentoring relationships with system-involved youth. Journal of Adolescent Research, DOI: 0743558418755686. 
Thompson, A. E., Greeson, J. K., \& Brunsink, A. M. (2016). Natural mentoring among older youth in and aging out of foster care: A systematic review. Children and Youth Services Review, 61, 40-50.

Unrau, Y. A., Seita, J. R., \& Putney, K. S. (2008). Former foster youth remember multiple placement moves: A journey of loss and hope. Children and Youth Services Review, 30, 1256-1266.

U. S. Department of Health and Human Services (HHS), Administration for Children, Youth, and Families. (2012). Information memorandum: Promoting social and emotional well-being for children and youth receiving child welfare services (Publication No. ACYF-CB-IM-12-04). Washington, DC: Author.

U.S. Department of Health and Human Services (HHS), Administration for Children and Families, Children's Bureau. The AFCARS Report: Preliminary FY 2016 Estimates as of October 20, 2017. US Department of Health and Human Services, Washington, DC. Retrieved from https://www.acf.hhs.gov/sites/default/files/cb/afcarsreport24.pdf

Webber, M., \& Fendt-Newlin, M. (2017). A review of social participation interventions for people with mental health problems. Social Psychiatry and Psychiatric Epidemiology, 52(4), 369-380.

Wellman, B., \& Frank, K. (2001). Network capital in a multilevel world: Getting support from personal communities. Social Capital: Theory and Research, 233-273.

Wellman, B., \& Wortley, S. (1990). Different strokes from different folks: Community ties and social support. American Journal of Sociology, 96(3), 558-588.

Wulczyn, F., Kogan, J., \& Harden, B. J. (2003). Placement stability and movement trajectories. Social Service Review, 77, 212-236.

Yates, T. M., \& Grey, I. K. (2012). Adapting to aging out: Profiles of risk and resilience among emancipated foster youth. Development and Psychopathology, 24(2), 475-492.

Zinn, A., Palmer, A. N., \& Nam, E. (2017). Developmental heterogeneity of perceived social support among former foster youth. Children and Youth Services Review, 76, 51-58. 


\section{Highlights}

- We conducted support network mapping and interviews with foster youth ( $N=22)$.

- Theoretical thematic analysis explored barriers and facilitators to support.

- Support capacity was limited by interpersonal difficulties between youth and others.

- Network stability facilitated support through strong, interconnected relationships.

- Emergent patterns reflected subgroups of more and less functional support networks. 